\title{
Application of Permanent Magnetic Material in Vibration Drilling
}

\author{
Wang Yao ${ }^{1}$, Zhang Qijiu ${ }^{1}$, Zhang Zejian" ${ }^{1}$, Bai Yan ${ }^{2, *}$ \\ ${ }^{1}$ School of Mechanical Engineering, Beihua University, Jilin, China \\ ${ }^{2}$ Engineering Training Center, Beihua University, Jilin, China \\ *Corresponding author
}

Keywords: vibration drilling, permanent magnetic material, vibration exciter

\begin{abstract}
A method based on vibration cutting is proposed to overcome the difficulty of chip removal for small diameter deep hole drilling. The method of vibration drilling has the advantages of small drilling force, high drilling accuracy and high chip removal performance.
\end{abstract}

\section{Introduction}

Permanent magnet materials, as the coils, have the characteristics of establishing a magnetic field [1]. But the biggest advantage of the permanent magnetic material is that once the magnetic field is established, there is no energy consumption, no coil like power supply equipment, no heating phenomenon, and stable, small volume [2][3]. So its application is very extensive. A lot of vibration drilling incentives, through the design of permanent magnetic materials and magnetic circuit shape made of permanent magnet vibration exciter is reasonable, can make the principal axes of attraction and repulsion alternately in the production process, and reply to effect by spring force so that the spindle vibration amplitude, can be adjusted through regulating the gap size and magnetic circuit change the repulsion and suction to achieve, vibration frequency adjustment can be realized by changing the speed of the spindle or number of vibrations per turn [4].

\section{Performance Requirements of Permanent Magnet Vibration Drilling for Permanent Magnetic Material}

Application of permanent magnetic material, but to sum up, according to the working state of the permanent magnet can be divided into static (solid flux air gap or air gap constant) and dynamic (flux variation of air gap or air gap in two). Because the amplitude of the vibration drilling is very small, it can be recognized that the magnetic circuit air gap is fixed, so the requirements of the permanent magnetic material under static conditions are mainly introduced below. In this case, is to produce a certain magnetic field strength in a given space by using permanent magnetic materials, because the close loop of permanent magnet external performance of the effective energy is zero, can not meet the use requirements, so the circuit cannot be closed, but open, the air gap has a certain gap. In the case of open circuit, there is a free magnetic pole at both ends of the material, which will result in a demagnetizing field $\mathrm{Hd}$. The direction of the demagnetizing field is in opposition to the magnetic induction in the material (Hd=-NM). Thus, the magnetic remanence shown in fact is not $\mathrm{Br}$, but rather decreases to a point in the second quadrant demagnetization curve, such as the magnetic induction intensity corresponding to the $\mathrm{D}$ point(Bd), as shown in figure 1 .

The best working point and maximum magnetic energy product of permanent magnetic material can be obtained by geometrical drawing of demagnetization curve, as shown in figure 2. Practice has proved that the material $(\mathrm{BH})$ and the size of materials $\mathrm{Max} \mathrm{Br}, \mathrm{Hc}$, and the demagnetization curves of different shapes, in the same load line (under the same angle) the corresponding working point of $\mathrm{B}, \mathrm{H}$ values are not the same, as shown in figure 3 . It can be seen from the diagram that the demagnetization curve is more prominent, and the corresponding $\mathrm{B}$ and $\mathrm{H}$ are larger, so the corresponding magnetic energy product is the largest. 


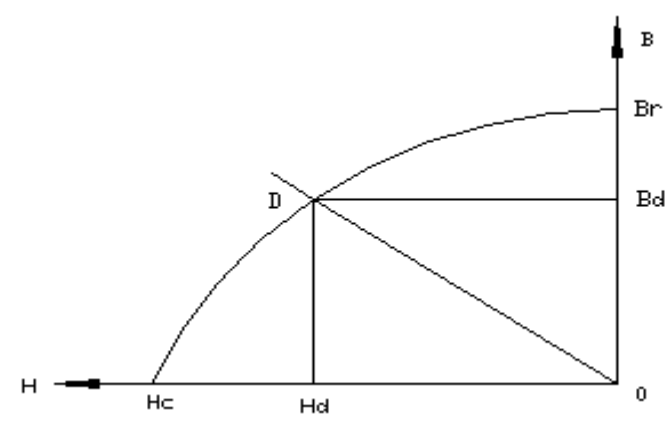

Fig.1 Working point of permanent magnet

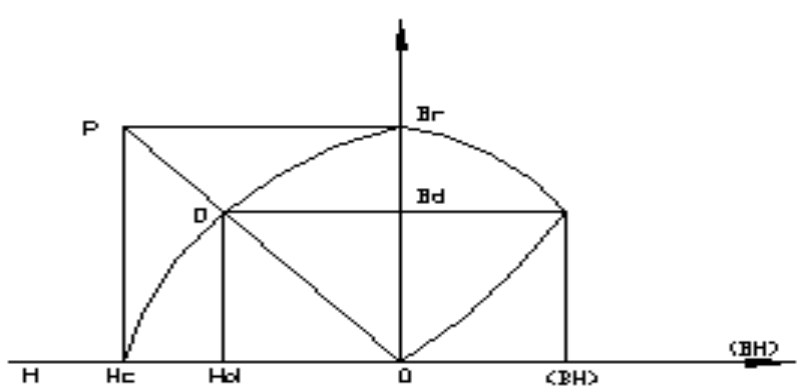

Fig.2 graphing method for the maximum energy product

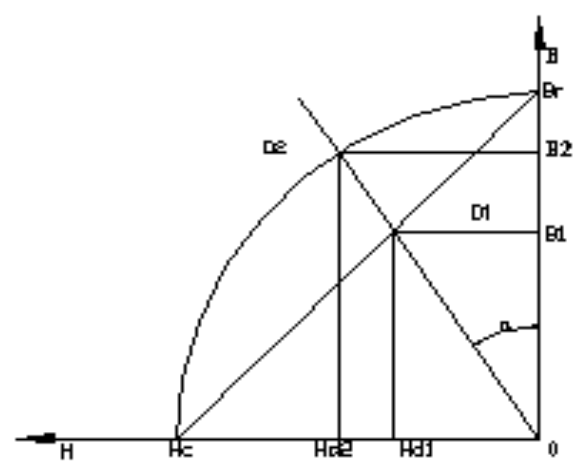

Fig.3 Influence of demagnetization line shape on working point

\section{Design of Permanent Magnet Exciter}

At work, there is an air gap between the two plates, and the rotating disk rotates with the main shaft at a speed of $n$. In this way, all the magnetic poles on the static disk simultaneously attract or repel the moving disk periodically, and cause the axial vibration of the main shaft under the restoring force of the spring. Each pass through two magnetic poles, the main shaft to complete a vibration. Each spindle rotates five times in axial direction. Generally, the number of the magnetic pole is $\mathrm{m}$, and the spindle vibration frequency is $\mathrm{P}=\mathrm{mn} / 120$ (HZ). When the speed is constant, the more the magnetic pole, the higher the vibration frequency; and the vibration frequency increases with the increase of revolution. In the open case, due to the demagnetizing field, will enable the permanent magnetic material as decreases in magnetic induction intensity, and the air gap length LG is larger, the demagnetizing field strength increases, the permanent magnetic magnetic material utilization rate is low, the magnetic force is small, therefore, the amplitude of permanent magnet vibration exciter has the smaller .The machine tool feed motion system and permanent magnet exciter is shown in the figure 4 and figure 5.

Above all, the permanent magnet exciter, can change the speed of $\mathrm{N}$ or change the poles to adjust the vibration frequency of P; by changing the air gap size of LG to adjust the amplitude, at the same time with the cutting amount change, can be adjusted to the optimum parameters of vibration drilling, drilling to reach the best effect. 


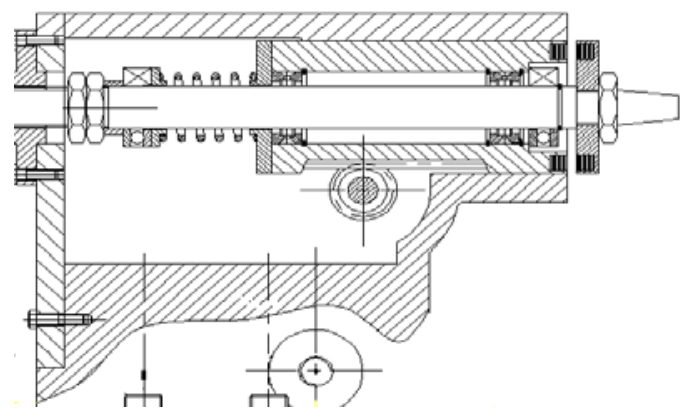

Fig.4 Machine tool feed motion system

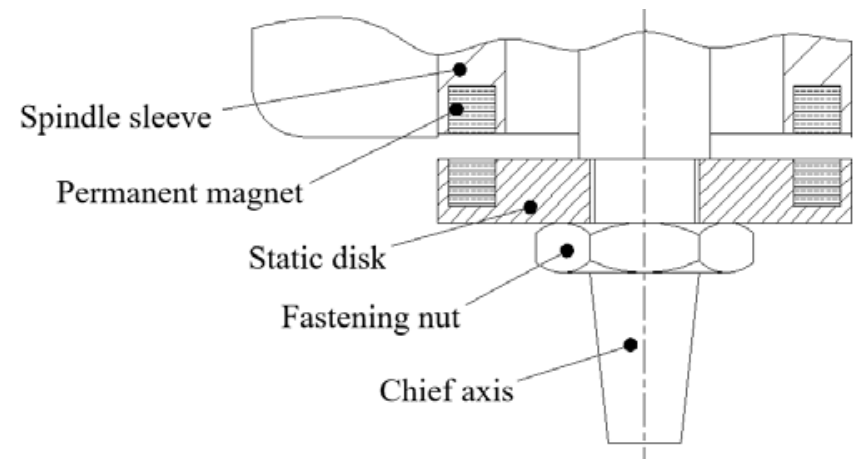

Fig.5 Permanent magnet exciter

\section{Vibration Waveform of Permanent Magnet Exciter}

For the convenience of analysis, take two permanent magnets, for example, the other side of the panel as the moving disk, relative to it clockwise rotation, the initial state is set to $\mathrm{N}$, the pole is very opposite to the $\mathrm{S}$.

(1) the initial two-disc phase, and the maximum suction;

(2) after 90 degrees, the suction force and repulsion force just cancel out, and the axial interaction force between the two plates is zero;

(3) turned 180 degrees, two-disc repulsion, and maximum repulsion;

(4) when rotating 270 degrees, the suction and repulsion are offset, and the axial interaction force between the two plates is zero;

(5) turned 360 degrees, back to the initial state, two phase, and the maximum suction.

Make two sets of suction negative, do two disk interaction force and moving disk angle of rotation curve, as shown in figure 6 . It can be seen that for the tile shaped permanent magnet, the exciting vibration of the two plates is cosine curve in the relative rotation of the two plate.

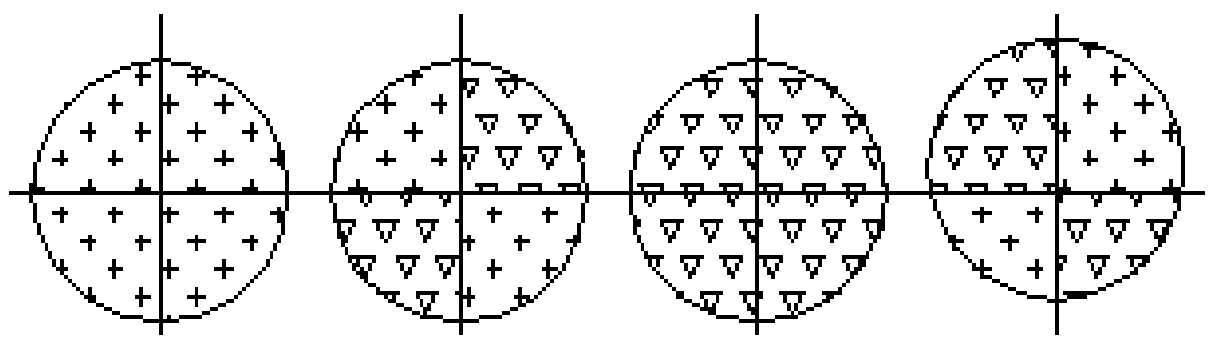

Fig.6 Schematic diagram of exciting force analysis

The circular pole, as shown in Figure 7, the permanent magnet is instantaneous area change, zero force time non instantaneous value, in addition, in the two relative rotation process, two opposite poles by the outer edge began to cut into the process, the area change is nonlinear so the excitation force waveform is not possible a sine or cosine curve, and the permanent magnets in the same length, the total volume of the whole tile shaped magnet product is greater than the circular pole, so the excitation force is larger. 


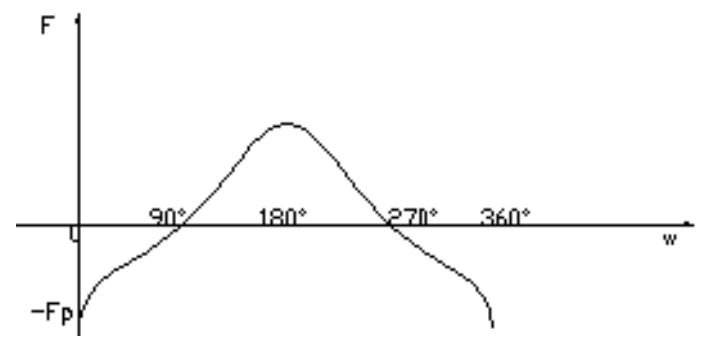

Fig.7 F- $\omega$ curve

\section{Conclusion}

In this paper, through the analysis of cutting performance requirements of permanent magnetic materials of permanent magnet vibration drilling, the permanent magnet in the maximum energy product corresponding to the operating point, magnetic energy of permanent magnet produced in the air gap on the direction of the product is $\mathrm{D}$ the maximum point is the optimum working point. Therefore, the design and analysis of the permanent magnet exciter are carried out in detail, and the vibration waveform is obtained with cosine waveform, which provides a theoretical basis for the subsequent perfect design.

\section{Acknowledgment}

The research is funded by the project development plan of science and technology in Jilin Province (20160418024FG).

\section{References}

[1] Wang X, Wang L J, Tao J P. Investigation on thrust in vibration drilling of fiber-reinforced plastics [J]. Journal of Materials Processing Tech, 2004, 148(2):239-244.

[2] Linbo Z, Lijiang W, Xin W. Study on vibration drilling of fiber reinforced plastics with hybrid variation parameters method[J]. Composites Part A, 2003, 34(3):237-244.

[3] Okamura K, Sasahara H, Toshiaki S, et al. Low-Frequency Vibration Drilling of Titanium Alloy [J]. Jsme International Journal, 2006, 49(1):76-82.

[4] Weinert K, Webber O, Peters C. On the Influence of Drilling Depth Dependent Modal Damping on Chatter Vibration in BTA Deep Hole Drilling[J]. CIRP Annals-Manufacturing Technology, 2005, 54(1):363-366. 